\title{
Location base Routing with Energy Awareness Scheme under Wi-Max in MANET for Minimization of Routing Overhead
}

\author{
MadhukarDubey \\ M.Tech Scholar, RKDFIST \\ College, Bhopal, India
}

\author{
Piyush Singh \\ Assistant Professor,Dept.of \\ Computer Science, \\ RKDFISTCollege,Bhopal,India
}

\author{
Gaurav Shrivastava \\ Head Dept. of Computer \\ Science, RKDFIST College, \\ Bhopal, India
}

\begin{abstract}
Mobile ad hoc network is a collection of wireless movable node. Movement of nodes results in a change in routes, requiringsome mechanism for determining new routes, Verity of routing protocol exist under MANETs for managing network but all existing routing technique spread maximal overhead so location aware base routing technique is applied that provide minimal overhead as compare to existing routing protocol, In mobile ad-hoc network energy constraint devicesare used the motive is to minimize required power need for data transmission and increase reliability of data delivery.In this paper minimizationof routing overhead is achieved which increasenetwork life time, save energy and increase packet delivery ratio. E-AODV-LAR approach is proposed whichprovide more reliable as well as minimum routing overhead, through results analysisis done on the bases of all network parameterand get efficient technique for MANETs environment.
\end{abstract}

\section{Keywords}

MANETs, Energy, LAR, AODV, Wi-Max, Packet Delivery Ratio, Died Node

\section{INTRODUCTION}

Wireless networks can be classified into two categories: infrastructure mobile networks and non-infrastructure mobile networks. MANET (Mobile Ad hoc Network) belongs to the latter category. There are no dedicated routers, or servers. It is formed by a set of moving nodes. Every mobile node (MN) can be a router and the packet transmission is a multi-hop.

Many routing protocols are developed for discovering and recovering the communication paths among mobile nodes. They can be separated into two categories: connection-oriented or connectionless. It depends on whether a connection, i.e., route, discovered or not for data transmission. Most of the MANET routing protocols are connection-oriented since it is the basic principle of data transmission on Internet. Number of routing protocol applies for on demand bases routing protocol for route search like AODV, DSR etc.

Considering the challenging and demanding aspects by the modern wireless systems, the broadband wireless access industry, which provides high-rate network connections to stationary locations and end stations, has advanced to a point at which it now has a robust standard for second generation wireless metropolitan area networks. IEEE 802.16 standard which is well known as worldwide interoperability for microwave access (Wi-MAX) is the solution for such wireless networks [1]. When this technology is considered for mobile networks, it is expected to provide around $15 \mathrm{Mbps}$ of channel capacity within a particular cell.

The paper organization is as follows: Section 2 describes the related work, in section 3 Routing protocols AODV and location aided routing is described, in section 4objective is mentioned. The problem statement, proposed solution and modified AODV according to proposed algorithm are described in section 5,6 and 7. Network simulation with results are presented in section 8 followed by conclusions and future work in section 9 .

\section{RELATED WORK}

Several studies have dealt with measuring energy consumption in the wireless interfaces of mobile nodes, e.g.

Ali-Yahiya et al. [2] propose an architecture where the Wi-Fi and Wi-MAX networks and their traffic routes are separated by dedicated gateways to provide interconnectivity. The main characteristic of this architecture is that it employs an overlapping area between a WLAN and a WMAN hotspot, where they interconnect at BS and AP. The WMAN coverage area expands using the WLAN hotspots. More than one AP can be located near the limit of the WMAN's coverage area. The authors assume that Wi-Fi network APs are connected to the Wi-MAX network by means of a gateway that permits bidirectional interconnectivity.

Ntlatlapa in [3] focused on the potential of the IEEE 802.16 mesh mode and proposed a routing algorithm that is suitable for coordinated distributed scheduling. The algorithm presented is an adaptation of a simple approach to routing called Better Approach to Mobile ad hoc Networking (BATMAN) for 802.16 wireless mesh networks.

Nivedita N. Joshi et al. proposed "Energy Conservation In MANET Using Variable Range Location Aided Routing Protocol" [4] Their proposed protocol is known as Variable Range Energy aware Location Aided Routing (ELAR1-VAR). The proposed scheme controls the transmission power of a node according to the distance between the nodes. It also includes energy information on route request packet and selects the energy efficient path to route data packets.

Neelesh Gupta et al proposed "Optimization of Performance Metrics of LAR in Ad-Hoc Network" [5].In this paper, developments of performance-metrics using LAR have been reported. Using these developments, LAR protocol has been found to be better than other protocols like DSR in MANETs of different scenario. This is because of already available location information of the nodes in network while it is based upon source routing as a DSR.

Young-BaeKo and Nitin H. Vaidya has proposed "LocationAided Routing (LAR) in Mobile Ad Hoc Networks" [6] This paper suggests an approach to utilize location information (for 
instance, obtained using the global positioning system) to improve performance of routing protocols for ad hoc networks. By using location information, the proposed Location-Aided Routing (LAR) protocols limit the search for a new route to a smaller "request zone" of the ad hoc network. This results in a significant reduction in the number of routing messages.

\section{ROUTING PROTOCOL FOR FINDING LOCATION AND ROUTING}

\subsection{Location-Aided Routing Protocol}

Location-Aided Routing (LAR) [7][8] is an on-demand routing protocol which exploits location information. In fact, LAR operates very similarly to DSR. The major difference between the two protocols is that LAR uses location information obtained from GPS to restrict the flooded area of Route Request packets.

There are two schemes to determine which nodes propagate Route Requests. In scheme 1, the source defines a circular area in which the destination may be located. The position and size of the circle is decided with the following information:

(a) The destination location known to the source;

(b) The time instant when the destination was located at that position; and

(c) The average moving speed of the destination.

The smallest rectangular area that includes this circle and the source is the request zone. This information is attached to a Route Request by the source and only nodes inside the request zone propagate the packet. In scheme 2 , the source calculates the distance between the destination and itself. This distance, along with the destination location known to the source, is included in a Route Request and sent to neighbours. When nodes receive this packet, they compute their distance to the destination, and continue to relay the packet only if their distance to destination is less than or equal to the distance indicated by the packet. When forwarding the packet, the node updates the distance field with its distance to the destination. In both schemes, if no Route Reply is received within the timeout period, the source retransmits a Route Request via pure flooding.

\subsection{Ad-Hoc On Demand Distance Vector}

An ad hoc network is the cooperative engagement of a collection of mobile nodes without the required intervention of any centralized access point or existing infrastructure. AODV [9] is a novel algorithm for the operation of such ad hoc networks. Each mobile host operates as a specialized router, and routes are obtained as needed (i.e., on demand with little or no reliance on periodic advertisements). The AODV routing algorithm is quite suitable for a dynamic self-starting network as required by users wishing to utilize ad hoc networks. AODV provides loop-free routes even while repairing broken links. Because the protocol does not require global periodic routing advertisements, the demand on the overall bandwidth available to the mobile nodes is substantially less than in those protocols that do necessitate such advertisements.

\section{OBJECTIVE}

The objective is to utilize energy of each node with efficient way under WIMAX environment with AODV routing protocol and also minimize routing overhead with the LAR (location aware routing) through thisthe following goals are achieved:
$>$ It is providing low routing overhead because LAR gives location information from source to destination path, if routing change in certain condition.

$>$ More throughputs are achieved from existing network, because WIMAX provides maximum radio range and low power consumption.

$>$ Analysis and awareness about each node is achieved through energy awareness routing, for prolonging the network life time the energy level of each node is balanced according to the load.

$>$ It is also minimizing data drop from the network and increase packet delivery ratio of the network.

\section{PROBLEM STATEMENT}

Now a day MANETs is avital and really challenging research field because of its unpredictability. In this each node of the network is capable of routing and they can dynamically move anywhere without having any central controlling system. In mobile ad-hoc network each and every movement of the nodeschanges routing table which also upgrades the network topology every time, and very importantlymobile nodes run on the limited power source so a system is proposed to minimize the routing overhead and the efficient utilization of energy for data transmission under WIMAX in MANET. All theanalysis and the simulation are done in the network simulator-2.

\section{PROPOSED SOLUTION}

In this work the way for minimization of routing overhead under MANETs is achieved which is a big challenge because ofdynamically changing topology. So Location based routing under AODV routingis proposed, in that case very first broadcast routing packet is delivered to all neighbours for finding out the destination node, after the destination node is discovered actual data will be transmitted, but after certain time network topology changes and destination becomes unreachable then recursively routing packet broadcast starts again, but inthis new approach for minimization of routing packet in MANETsLAR (location aware routing) is used that provide the location information of the destination and all existing path. so sender node uses that LAR packet and send routing packet to given direction and routing overhead will get minimized, by doing this routing overhead is minimizedbut not provide maximum reliability because if exiting routed node contain certain level of energy and it willdiminish in some time so in that case energy based routing is proposed which check each node energy and gives the information to the sender this module is called energy module both the above approaches are suitable for MANET and minimize routing overhead and maximize data delivery percentage. After that simulation scenario is created through TCL (tool command language) firstly mobile nodes are created and set routing protocol as AODV (ad-hoc on demand distance vector routing) after this LAR routing is applied for finding the expected zone of the destination. Then set the channel type as wireless channel, prorogation type two ray ground wave because mobile node contain routing table and also node radio range is limited so our data transmitted from node to node after that MAC ( media access control technique) is applied as $802.16 \mathrm{Wi}$-max that provides greater radio range as compare to 802.11 WLAN scheme.

\section{PROPOSED ALGORITHM}

An algorithm is designed for Energy with location aware routing under Wi-MAX standard in MANETs, in this algorithm firstly energy module with energy parameters are 
created like transmission, receiving, sleeping and sensing power with initial energy method, energy discharge formula and route call on the bases of energy after that in internal module location aware routing is added that provide estimated location of destination to the destination. in external TCL (tool command language) very first initial network parameter is set like physical parameter, MAC protocol channel type, antenna type and routing protocol after that mobile nodes are created with sender and receiver node and initial energy after thatroute function call is computed and discover route from source to destination on the bases of energy value but only that module case if route break in certain time so route function repetitive call and broadcast route packet that increases route overhead so LAR (location aware routing) is used that module is very useful for route overhead minimization, Inthis case receiver node send location information to the sender node time to time manner and useful for route discovery process and route packet flood only expected zone on the bases of previous location table, here algorithm is deployed step by step as follows:

Mobile node $=\mathrm{M} ; / /$ Number of mobile nodes

Sender node $=\mathrm{S} ; / /$ sub set of $\mathrm{M}$

Receiver Node $=\mathrm{R}$; //sub Set of M

Receiver Routing = LAR; // Location aware routing

Start simulation time $=\mathrm{t}_{0}$

Set routing protocol $=\mathrm{AODV}$;

Set $\mathrm{MAC}=802.16$

Set initial energy $=100$ joule

Set radio range $=\mathrm{rr}$; //initialize radio range

RREQ_B(S, R, rr)

\{

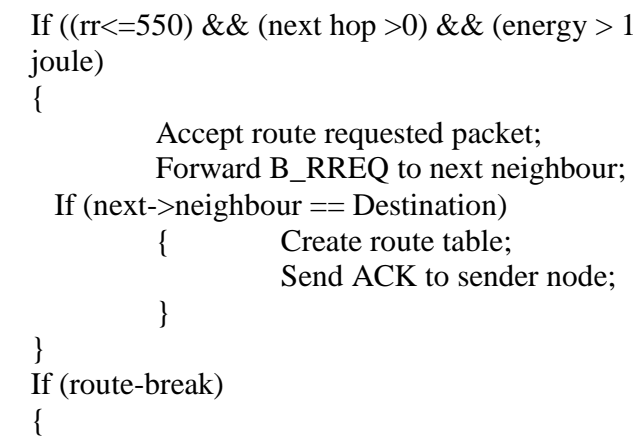

Accept route requested packet;

Forward B_RREQ to next neighbour; If (next->neighbour $==$ Destination)

$\{\quad$ Create route table;

Send ACK to sender node;

\}

If (route-break)

\{

Receiver R uses LAR routing; information to sender:

Receiver send expected location and speed

If (node updates location)

\{

Use location info and sender send routing packet to expected zone;

If $($ dest $==$ true $)$

\{

nexthop, type)

Data_packet_send(s_no,

\})

$$
\text { else }\{\text { destination not found; }
$$$$
\text { \} }
$$

else $\{$ destination un-reachable or energy value is less than 1 joule;

\} \}

\section{SIMULATION ENVIRONMENT AND RESULTS}

The simulation described in this paper was tested using the ns2 test-bed that allows users to create arbitrary network topologies [10]. By changing the logical topology of the network, ns-2 users can conduct tests in an ad hoc network without having to physically move the nodes. NS-2 controls the test scenarios through a wireless interface, while the ad hoc nodes communicate through a wireless interface. Here some essential simulation parameters are taken. The results are calculated on the basis of these parameters.

Simulation parameters to make the scenario of routing protocols in this work are as follows:

\section{TABLE 1: SIMULATION PARAMETERS FOR CASE STUDY}

\begin{tabular}{|l|l|}
\hline Number of nodes & 50 \\
\hline Dimension of simulated area & $800 \times 600$ \\
\hline Initial node energy (joules) & Random \\
\hline Threshold value(joule) & 1 \\
\hline Routing Protocol & AODV, LAR \\
\hline MAC & 802.16 \\
\hline Simulation time (sec) & 100 \\
\hline Radio range & $550 \mathrm{~m}$ \\
\hline Traffic type & CBR \\
\hline Packet size (bytes) & 512 \\
\hline Number of traffic connections & 30 \\
\hline Maximum Speed (m/s) & Random \\
\hline Node movement & random \\
\hline Tx energy consumption & $1.5 \mathrm{~J}$ \\
\hline Rx energy consumption & $1.0 \mathrm{~J}$ \\
\hline Idle energy consumption & $0.017 \mathrm{~J}$ \\
\hline Sleeping energy consumption & $0.001 \mathrm{~J}$ \\
\hline
\end{tabular}

\subsection{Scenario for Connection Establishment}

Here the snap shot of fifty nodes are shown they are sensing the neighbour for establish connection between the source nodes to destination node through intermediate nodes. In this scenario the green colour nodes are having a sufficient energy for communication. The scenario is created through Network Animator and shows initial node position in this architecture node motion is analysed as well as sender and receiver node communication.

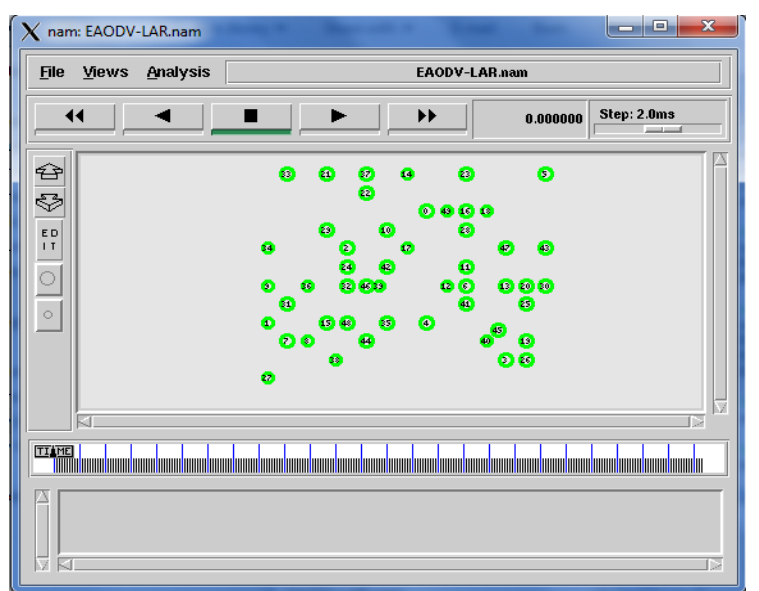

Figure1:Nodes are sensing their neighbour for establish connection. 


\subsection{Packet Delivery Ratio Analysis}

In this simulation fifty mobile nodes are created and calculate packet delivery ratio, packet delivery ratio is a ratio between packets receives by the authentic receiver from genuine packets sends by sender at current time.

$$
P D F=\left(\frac{R x}{\text { Send }}\right) * 100
$$

If packet delivery ratio is higher that means performance is best, here in this result packet delivery ratio (PDF) of E-AODV LAR scheme is greater than E-AODV scheme. It indicates that the E-AODV LAR scheme is more efficient than the E-AODV scheme.

In graph $\mathrm{x}$-axis is represented as a simulation time in seconds and $\mathrm{y}$-axis shows percentage of data delivery, this approachgives nearly $92 \%$ of data delivery that is nearly $3 \%$ greater than E-AODV.

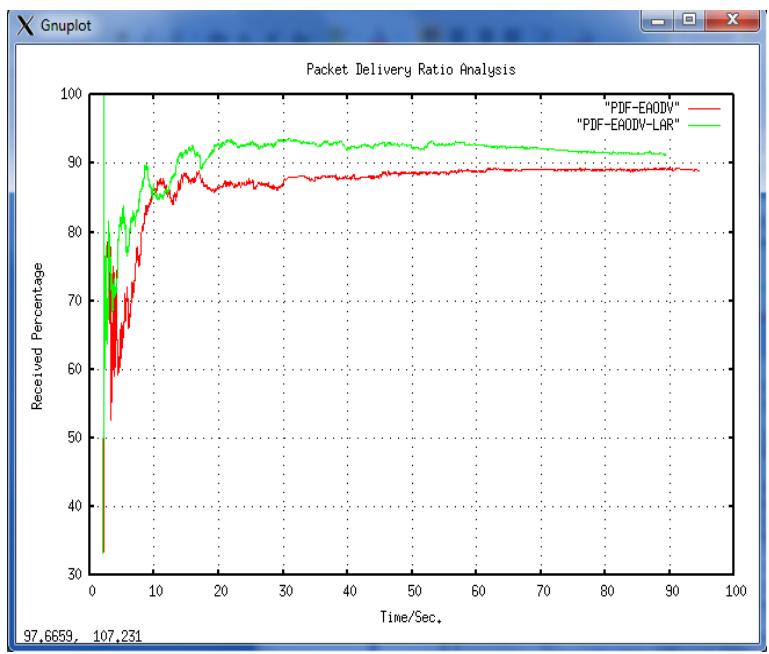

Figure 2: Packet Delivery Ratio Analysis

\subsection{Routing Load Analysis}

Routing load is calculated as the total number routing packets are transmitted over the successful data transmission. The increase in the routing load reduces the performance of the adhoc network as it consumes portions from the bandwidth available to transfer data between the nodes. Here E-AODV routing overhead is more than 12000 while E-AODV LAR overhead is only 4100 approximately. As per result E-AODV transmits more routing packets as compared to E-AODV LAR scheme. This result shows E-AODV LAR scheme is more efficient than E-AODV scheme.

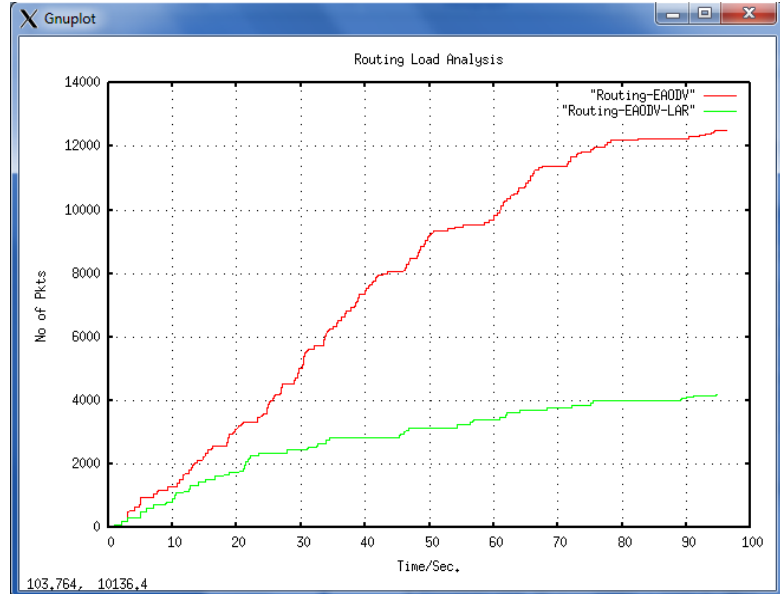

Figure 3: Scenario of routing load analysis.

\subsection{Died Node Analysis}

Here died node is analysed in network with energy base AODV and LAR proposed technique, that graph shows in $\mathrm{x}$-axis is number of nodes and $y$-axis shows died time, died node means node energy are zero (existence in network but not work because energy is zero). Results concluded that died node are minimum as compare to E-AODV because E-AODV-LAR minimize routing flooding and increases life time of the network.

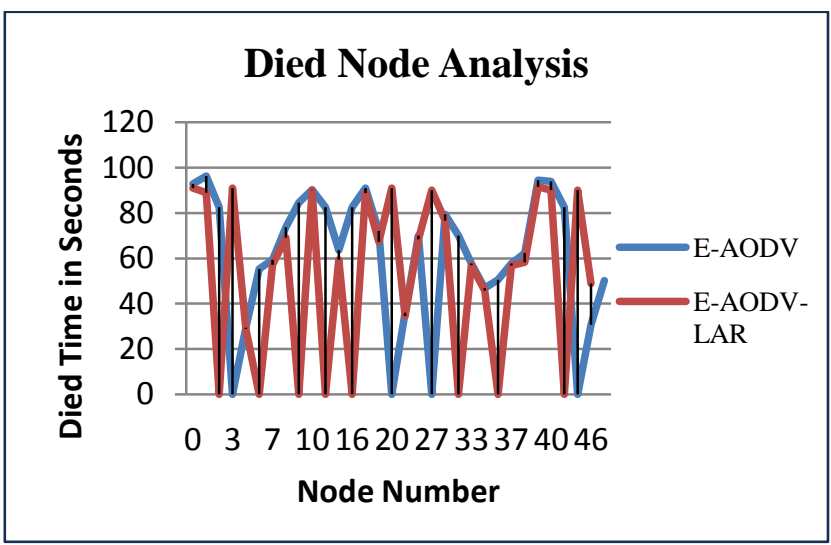

Figure 4: Died node Analysis

\subsection{Remain Energy Analysis}

Below the remain energy is retrieved of each node, for that result conclude energy saving after simulation time end, table no. 2 shows E-AODV-LAR technique which save nearly 37 joule energy which is greater as compare to E-AODV technique, in energy base approach case discharge energy is applied on the bases of transmission, receiving, sleeping and ideal power base.

Remaining energy of the whole network is calculated by using following formula:

\section{Discharge energy \\ $=(($ TxP or RxP or IdealP or SleepP $) *$ Time taken}


Table 2: Results of remaining Energy of each node usingE-AODVand E-AODV-LAR techniques

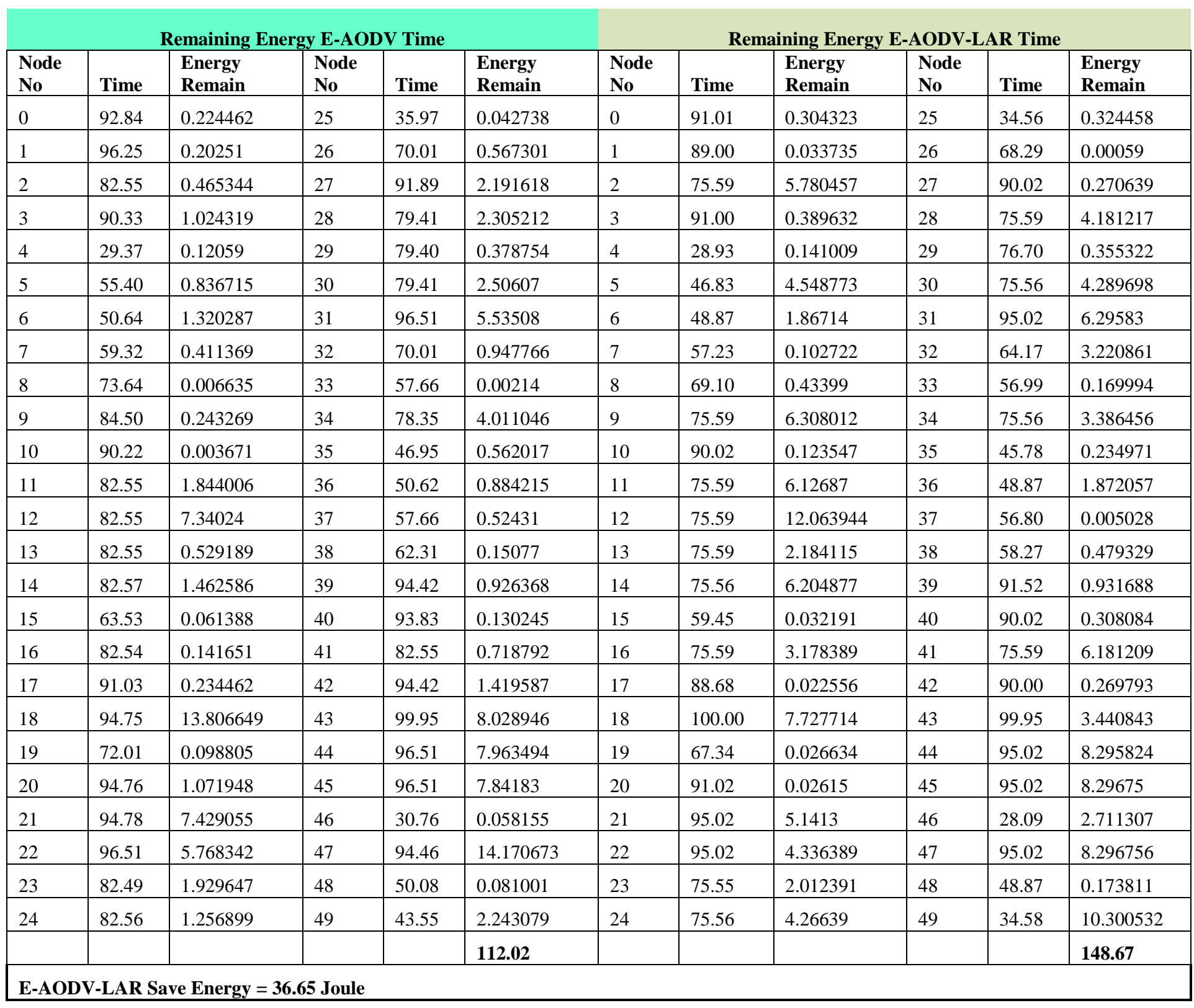

\section{CONCLUSION AND FUTURE WORK}

This strategy mainly analyzes the network behavior under location awareness as well as energy base routing scheme under Wi-max environment.

Here NS-2 is used as simulator and tested the working multiple simulation base it is concluded that the proposed work increases packet delivery ratio as well as throughput of the network with minimum routing load, it also uses less energy as compare to energy base AODV routing. Finally it is concluded that location base routing provide lower overhead and energy aware approach which provide reliable and efficient communication.

In this simulation is done through energy aware with location base routing schemes under Wi-max, the results are simulated through NS-2 and use routing protocol as AODV and LAR, In future this concept is applied with all different MANET protocols like DSR, DSDV and OLSR with LAR scheme and find out total routing overhead minimization.
In future various energy depletion parameters will used and simulate the work which rectified the result get from theproposedmodule.

\section{ACKNOWLEDGMENTS}

It is my immense pleasure to express my deep sense of gratitude and indebtedness to my highly respected and esteemed guide Mr.Piyush Singh, Assistant Professor and Mr. K.K. Puranik, Director R.K.D.F.I.S.T, Bhopal. Their invaluable guidance, inspiration, constant encouragement sincere criticism and sympathetic attitude could make this dissertation possible.

I extend my deep sense of gratitude to Mr.GauravShrivastava, Head of the Department of Computer Science and Engineering, R.K.D.F. Institute of Science \& Technology, Bhopal for providing encouragement, guidance and support during my work. I extend my thanks to all the staff members of the department who extended their co-operation and help throughout the work. 


\section{REFERENCES}

[1] IEEE 802.16-2001, Apr. 2002, IEEE Standard for Local and Metropolitan Area Networks - Part 16: Air Interface for Fixed Broadband Wireless Access Systems.

[2] T. Ali-Yahija, K. Sethom, y G. Puiolle, Proc. 2007, Seamless Continuity of Service across WLAN and WMAN Networks: Challenges and Performance Evaluation, IEEE/IFIP International Workshop, pp. 1-12

[3] Ntlatlapa, N. 2008, A Routing Metric and Algorithm for IEEE802.16 Mesh Networks, BROADCOM, pp: 324 - 328.

[4] Nivedita N. Joshi and Radhika D. Joshi, , October 2011, Energy Conservation in MANET Using Variable Range Location Aided Routing Protocol, International Journal of Wireless \& Mobile Networks (IJWMN) Vol. 3, No. 5.

[5] Neelesh Gupta and Roopam Gupta, June 2012, Optimization of Performance Metrics of LAR in Ad-Hoc Network, International Journal of Wireless \& Mobile Networks (IJWMN) Vol. 4, No. 3.
[6] Young-BaeKo and Nitin H. Vaidya, Location-Aided Routing (LAR) in Mobile Ad Hoc Networks, Research reported is supported in part by Texas Advanced Technology Programgrants 010115-248 and 009741-052-C

[7] Y. B. Ko AND N.H. VAIDYA, OCTOBER 1998, LOCATIONAIDED ROUTING (LAR) IN MOBILE AD HOC NETWORKS, PROCEEDINGS OF THE ACM/IEEE INTERNATIONAL CONFERENCE ON MOBILE COMPUTING AND NETWORKING (MOBICOM), DALLAS, TX, PP. 66-75.

[8] Mauve M. Widmer A. and Hartenstein H. "A Survey on Position-Based Routing in Mobile Ad-Hoc Networks" IEEE Network, vol. 15, Issue 6, pp.30-39, Nov.2001.

[9] Gupta N. and Gupta R., "Routing Protocols in Mobile AdHoc Network: An Overview" Proc.International Conference on Emerging Trends in Robotics and Communication Technologies(INTERACT), IEEE Explore pp. 173-177, 3-5 Dec., Chennai, 2010.

[10] The NetworkSimulator-ns-2 www.isi.edu/nsnam/ns 